Pacific Journal of Mathematic 


\section{THE REPRESENTATION OF AN ANALYTIC FUNCTION BY GENERAL LAGUERRE SERIES}

\section{OtTo SzÁsz AND NeLson Yeardley}

1. Introduction. Hille [4] has solved the problem of finding necessary and sufficient conditions that a function be represented by Hermitian series in a strip. Pollard [7] has solved the analogous problem in a strip for Laguerre series of order zero. We propose to solve the problem for Laguerre series of order $\alpha(\alpha>-1)$ getting as a region of convergence a parabola instead of a strip. From this theorem the generalization of Pollard's result follows immediately.

We say that a function of a complex variable $f(z)$ where $z=x+i y=$ $r e^{i \theta}$ possesses a Laguerre series of order $\alpha(\alpha>-1)$ or a general Laguerre series if

$$
f(z) \sim \sum_{n=0}^{\infty} \alpha_{n}^{(\alpha)} L_{n}^{(\alpha)}(z) \quad(n=0,1,2, \cdots)
$$

where

$$
a_{n}^{(\alpha)}=\left\{\left(\begin{array}{c}
n+\alpha \\
n
\end{array}\right) \Gamma(\alpha+1)\right\}^{-1} \int_{0}^{\infty} e^{-x} x^{x} L_{n}^{(\alpha)}(x) f(x) d x \quad(\alpha>-1)
$$

$L_{n}^{(\alpha)}(x)$ is the Laguerre polynomial of order $\alpha>-1$ and degree $n$ given by [ 8 p. 97 formula 5.1.6] and the above series converges. The series is said to be the Laguerre expansion of $f(z)$.

We define

$$
d_{\alpha} \equiv-\lim _{n} \sup \left(2 n^{\frac{1}{2}}\right)^{-1} \log \left|a_{n}^{\alpha}\right|
$$

and by the notation

$$
z \in p(b) \quad b>0 ; \quad z \in \bar{p}(b)
$$

we mean respectively that $z$ lies in the open (closed) parabolic region

$$
p(b): y^{2}<4 b^{2}\left(x+b^{2}\right) ; \quad \bar{p}(b): y^{2} \leqq 4 b^{2}\left(x+b^{2}\right) .
$$

If we select that branch of $z^{\frac{1}{2}}$ for which $(-z)^{\frac{1}{2}}$ is real and positive when $z<0$ then $\Re(-z)^{\frac{1}{2}}=\left\{\frac{1}{2}(r-x)\right\}^{\frac{1}{2}}=b^{2}$ gives the equation $y^{2}=$ $4 b^{2}\left(x+b^{2}\right)$ of the parabola which is the boundary of the above regions.

The main result of this paper is the following.

THEOREM A. In order that the function $f(z)$ possess a Laguerre series of order $\alpha(\alpha>-1)$ (or a general Laguerre series) which converges to it

Received March 28, 1958. 
for $z \in p\left(d_{\alpha}\right)$ where $d_{\alpha}>0$ (i.e. for every point $z$ lying in the parabola $\left.y^{2}=4 d_{\alpha}^{2}\left(x+d_{\alpha}^{2}\right)\right)$ it is necessary and sufficient that $f(z)$ be analyiic for $z \in p\left(d_{\alpha}\right)$ and that to every $b_{\alpha}$ such that $0 \leqq b_{\alpha}<d_{\alpha}$ there is a positive number $B(\alpha, b)$ such that

$$
|f(z)| \leqq B(\alpha, b) \exp \left\{\frac{1}{2} x-|x|^{\frac{1}{2}}\left[b_{2}^{\alpha}-\frac{1}{2}(r-x)\right]^{\frac{1}{2}}\right\} \quad\left(z \in \bar{p}\left(b_{\alpha}\right)\right) .
$$

2. The necessity of Theorem A. By hypothesis we have $d_{x}>0$ where $d_{\alpha}$ is given by (1.3) and

$$
f(z)=\sum_{n=0}^{\infty} \alpha_{n}^{(\alpha)} L_{n}^{(\alpha)}(z) \quad\left(z \in p\left(d_{\alpha}\right) ; \alpha>-1\right) .
$$

From Definition (1.3) it follows that

$$
\left|a_{n}^{(\alpha)}\right|<m(\alpha, \varepsilon) \exp \left\{2 n^{\frac{1}{2}}\left(-d_{\alpha}+\varepsilon\right)\right\} \quad(\varepsilon>0) .
$$

We use the estimates

$$
\left(\begin{array}{c}
n+\alpha \\
n
\end{array}\right) \sim n^{\alpha} / \Gamma(\alpha+1) \quad(n \rightarrow \infty ; \alpha \neq-1,-2, \cdots)
$$

and $\log n<(4 ! n)^{\frac{1}{2}}$. By defining

$$
c_{\alpha} \equiv \frac{1}{2}\left(b_{\alpha}+d_{\alpha}\right)>b_{x}
$$

and using the above estimate of $\left|a_{n}^{\alpha}\right|$ we can show that the series

$$
A^{2}(\alpha, b)=\sum_{n=0}^{\infty}\left|a_{n}^{(\alpha)}\right|^{2} \exp \left(4 c_{\alpha} n^{\frac{1}{2}}\right)\left(\begin{array}{c}
n+\alpha \\
n
\end{array}\right)
$$

converges $(\alpha>-1)$ by comparing it with the series $\sum_{n} 1 / n^{2}$.

By direct calculation we can verify that series (2.1) can be put in the form

(2.4) $f(z)=\sum_{n=0}^{\infty} a_{n}^{(\alpha)} \exp \left\{2 c_{x} n^{\frac{1}{2}}\right\}\left\{\left(\begin{array}{c}n+\alpha \\ n\end{array}\right)\right\}^{\frac{1}{2}} L_{n}^{(\alpha)}(z) \exp \left\{-2 c_{x} n^{\frac{1}{2}}\right\}\left\{\left(\begin{array}{c}n+\alpha \\ n\end{array}\right)\right\}^{-\frac{1}{2}}$

From the integral

$$
\exp \left(-s^{\frac{1}{2}}\right)=\left(1 / 2 \pi^{\frac{1}{2}}\right) \int_{0}^{\infty} e^{-s t} t^{-3 / 2} e^{-1 / t} d t
$$

we obtain

$$
\exp \left(-4 c_{\alpha} n^{\frac{1}{2}}\right)=\left(4 c_{\alpha} / 2 \pi^{\frac{1}{2}}\right) \int_{0}^{\infty} e^{-n q} q^{-3 / 2} \exp \left(-4 c_{\alpha}^{2} / q\right) d q .
$$

For polynomials $p_{n}(z)$ with real coefficients we have $\left|p_{n}(z)\right|^{2}=$ $p_{n}(\bar{z}) p_{n}(z)$.

By applying Cauchy's inequality the above two equations and [8 p. 98 formula 5.1.15] equation (2.4) becomes 


$$
\begin{aligned}
& |f(z)|^{2} \leqq\left[\sum_{n=0}^{\infty}\left|a_{n}^{(\alpha)}\right|^{2} \exp \left(4 c_{\alpha} n^{\frac{1}{2}}\right)\left(\begin{array}{c}
n+\alpha \\
n
\end{array}\right)\right] \\
& \times\left[\sum_{n=0}^{\infty}\left|L_{n}^{(\alpha)}(z)\right|^{2} \exp \left(-4 c_{\alpha} n^{\frac{1}{2}}\right)\left\{\left(\begin{array}{c}
n+\alpha \\
n
\end{array}\right)\right\}^{-1}\right] \\
& =A^{2}(\alpha, b) \sum_{n=0}^{\infty}\left|L_{n}^{(\alpha)}(z)\right|^{2}\left\{\left(\begin{array}{c}
n+\alpha \\
n
\end{array}\right)\right\}^{-1}\left(4 c_{\alpha} / 2 \pi^{\frac{1}{2}}\right) \int_{0}^{\infty} e^{-n t} t^{-3 / 2} \exp \left(-4 c_{\alpha}^{2} / t\right) d t \\
& =A^{2}(\alpha, b)\left(4 c_{\alpha} / 2 \pi^{\frac{1}{2}}\right) \int_{0}^{\infty}\left[\sum_{n=0}^{\infty}\left|L_{n}^{(\alpha)}(z)\right|^{2}\left\{\left(\begin{array}{c}
n+\alpha \\
n
\end{array}\right)\right\}^{-1} e^{-n t}\right] t^{-3 / 2} \exp \left(-4 c_{\alpha}^{2} / t\right) d t \\
& \left.=A^{2}(\alpha, b)\left(4 c_{\alpha} / 2 \pi^{\frac{1}{2}}\right) \int_{0}^{\infty} t^{-3 / 2} \exp \left(-4 c_{\alpha}^{2} / t\right)\left[\sum_{n=0}^{\infty} L_{n}^{(\alpha)}(z) L_{n}^{(\alpha)} \bar{z}\right)\left\{\left(\begin{array}{c}
n+\alpha \\
n
\end{array}\right)\right\}^{-1} e^{-n t}\right] d t \\
& =B(\alpha, b) \Gamma(\alpha+1) \int_{0}^{\infty} \frac{\exp \left(-2 x /\left(e^{t}-1\right)\right) J_{\alpha}\left(i|z| \operatorname{csch} \frac{1}{2} t\right) e^{\frac{1}{2} \alpha t}}{t^{3 / 2} \exp \left(4 c_{\alpha}^{2} / t\right)\left(1-e^{-t}\right)|z|^{\alpha} i^{\alpha}} d t
\end{aligned}
$$

for $z \in \bar{p}\left(b_{\alpha}\right), \alpha>-1$.

From [8 p. 197 formula (8.23.3)] we conclude that the following limit relation holds uniformly in any finite closed region $S$ in the complex $z-$ plane excluding the non-negative real axis

$$
n^{-\frac{1}{2}} \log \left|L_{n}^{(\alpha)}(z)\right| \rightarrow 2\left\{\frac{1}{2}(r-x)\right\}^{\frac{1}{2}}=2 r^{\frac{1}{2}} \sin \frac{1}{2} \theta \quad(n \rightarrow \infty) .
$$

We also have the estimate:

$$
\begin{aligned}
\left|L_{n}^{(\alpha)}(x)\right| & \leqq\{\Gamma(n+\alpha+1) / \Gamma(\alpha+1) \Gamma(n+1)\} \exp \left(\frac{1}{2} x\right) \quad(x \geqq 0) \\
L_{n}^{(\alpha)}(x) & =O\left(n^{a}\right) \quad a=\max \left(\frac{1}{2} \alpha-\frac{1}{4}, \alpha\right) \quad 0 \leqq x \leqq \omega
\end{aligned}
$$

and $\alpha$ is aribitrary and real. The later formula is found in [8 p. 173 formula (7.6.11)]. Moreover from [7 p. 85 formula 2] it follows that

$$
\Gamma(n+\alpha+1) / \Gamma(n+1) \sim n^{\alpha} \quad(n \rightarrow \infty) .
$$

By use of (2.6), (2.2) (2.7) (2.8) and the Weierstrass $M$-test to show uniform convergence of the above series and the comparison test to show convergence of the series in the second line of (2.5) we can then apply the theorem of [9 p. 44] to justify the term by term integration of the series in (2.5).

From [8 p. 15 formula (1.71.6)] and p. 14 formulas (1.7.4) and (1.7.5)] we obtain

$$
\left|J_{\alpha}(i \lambda) / i^{\alpha}\right|<\left(\frac{1}{2} y\right)^{\alpha} e^{\lambda} \mid \Gamma(\alpha+1) \quad\left(\alpha>-\frac{1}{2}, \lambda>0\right)
$$

from which we get by setting $\lambda=|z| \operatorname{csch} \frac{1}{2} t$

$\left|J_{\alpha}\left(i|z| \operatorname{csch} \frac{1}{2} t\right) i^{-\alpha}\right| \leqq\left(|z| \operatorname{csch} \frac{1}{2} t\right)^{\alpha} \exp \left(|z| \operatorname{csch} \frac{1}{2} t\right) 2^{-\alpha} \quad\left(\alpha>-\frac{1}{2}\right)$.

Using the above inequality to obtain an estimate of the last integral of (2.5) we obtain :

(2.9) $|f(z)|^{2} \leqq B(\alpha, b) 2^{-\alpha} \int_{0}^{\infty} \frac{\exp \left(-2 x /\left(e^{t}-1\right)+2|z| /\left(e^{\frac{1}{2} t}-e^{-\frac{1}{2} t}\right)\right)}{t^{3 / 2} \exp \left(4 c_{\alpha}^{2} / t\right)\left(1-e^{-t}\right)^{\alpha+1}} d t$. 
Since

$$
0 \leqq e^{\frac{1}{2} t}-e^{-\frac{1}{2} t} \geqq t
$$

we have

$$
\begin{aligned}
& -2 x /\left(e^{t}-1\right)+2|z| /\left(e^{\frac{1}{2} t}-e^{-\frac{1}{2} t}\right)-4 c_{\alpha}^{2} / t \\
= & 2 x\left(e^{\frac{1}{2} t}-1\right)\left(e^{\frac{1}{2} t}+1\right)\left(e^{\frac{1}{2} t}-1\right)+(2|z|-2 x) /\left(e^{\frac{1}{2} t}-e^{-\frac{1}{2} t}\right)-4 c_{\alpha}^{2} / t \\
\leqq & 2 x /\left(e^{\frac{1}{2} t}+1\right)-4\left\{c_{\alpha}^{2}-\frac{1}{2}(r-x)\right\} / t .
\end{aligned}
$$

If $q \equiv c_{\alpha}^{2}-\frac{1}{2}(r-x)$ then by applying the last inequality to (2.9) we obtain

$$
\begin{aligned}
& \left|f(z)^{2}\right| \leqq \int_{0}^{\infty} \exp \left(2 x /\left(e^{\frac{1}{2} t}+1\right)-4 q / t\right) t^{-3 / 2}\left(1-e^{-t}\right)^{-(x+1)} d t\left(\alpha>-\frac{1}{2}\right) \\
& \quad=B(\alpha, b) e^{x} \int_{0}^{\infty} \exp \left\{-x\left[1-2 /\left(e^{\frac{1}{2} t}+1\right)\right\} \exp (-4 q / t) t^{-3 / 2}\left(1-e^{-t}\right)^{-\alpha-1} d t .\right.
\end{aligned}
$$

Since $e^{\frac{1}{2} t}+1>2+\frac{1}{2} t(t \geqq 0)$ we get from the last integral

$$
\begin{aligned}
& |f(z)|^{2} \leqq B(\alpha, b) e^{x} \int_{0}^{\infty} \exp \{-x t /(t+4)\} \exp (-4 q / t) t^{-3 / 2}\left(1-e^{-t}\right)^{-x-1} d t \\
& \quad=e^{x}\left[\int_{0}^{16 q^{\frac{1}{2}}|x|^{\frac{1}{2}}}+\int_{16 q^{2}}^{\infty} \frac{1}{2} /|x|^{\frac{1}{2}}\right] \exp (-x t /(t+4)) \exp (-4 q / t) t^{-3 / 2}\left(1-e^{-t}\right)^{-\alpha-1} d t \\
& \quad=e^{x}\left(I_{1}+I_{2}\right) \quad\left(z \in \bar{p}\left(b_{\alpha}\right) ; \alpha>-\frac{1}{2}\right)
\end{aligned}
$$

where $I_{1}$ and $I_{2}$ represent the first and second integrals respectively in the above line. If $x>16 c_{\alpha}^{2}>16 q>0$ then

$$
-4 q^{\frac{1}{2}} x^{\frac{1}{2}} /\left(1+4 q^{\frac{1}{2}} x^{-\frac{1}{2}}\right)<-2 q^{\frac{1}{2}} x^{\frac{1}{2}}<-2 x^{\frac{1}{2} q^{\frac{1}{2}}}
$$

where $\tilde{q} \equiv b_{\alpha}^{2}-\frac{1}{2}(r-x)$. Since $\exp (-x t /(t+4))$ is a decreasing function of $t(t \geqq 0)$ we have

$$
\begin{aligned}
I_{2} \leqq \exp \left\{-x\left[16 q^{1 / 2}|x|^{-\frac{1}{2}} /\left(16 q^{\frac{1}{2}}|x|^{-\frac{1}{2}}+4\right)\right]\right\} \\
\quad \times \int_{16 q^{\frac{1}{2}} /|x|^{\frac{1}{2}}}^{\infty} \exp (-4 q \mid t) t^{-3 / 2}\left(1-e^{-t}\right)^{-x-1} d t .
\end{aligned}
$$

Since

$$
\left(1-e^{-t}\right)^{-1}<2 / t \quad(0 \leqq t \leqq 1)
$$

and by applying inequality (2.10) to the above estimate of $I_{2}$ we have

$$
\begin{aligned}
& I_{2} \leqq \exp \left(-2 x^{\frac{1}{2}} q^{\frac{1}{2}}\right)\left\{\Gamma(\alpha+3 / 2) / 4\left(c_{\alpha}^{2}-b_{\alpha}^{2}\right)^{\alpha+3 / 2}\right. \\
& \left.+4 \Gamma\left(\frac{1}{2}\right) / 4\left(c_{\alpha}^{2}-b_{\alpha}^{2}\right)^{1 / 2}\left(1-e^{-1}\right)^{\alpha+1}\right\}=B(\alpha, b) \exp \left(-2 x^{\frac{1}{2}} q^{\frac{1}{2}}\right) \\
& \left(z \in \bar{p}\left(b_{\alpha}\right) ; x>16 c_{\alpha}^{2}, \alpha>-\frac{1}{2}\right) .
\end{aligned}
$$


Consider the function $\mu(t)=x t /(t+4)+\overline{4 q} / t$. If we define

$$
\begin{aligned}
& \bar{c}_{\alpha}^{2} \equiv c_{\alpha}^{2}-\frac{1}{2}\left(c_{\alpha}^{2}-b_{\alpha}^{2}\right)=\frac{1}{2}\left(c_{\alpha}^{2}+b_{\alpha}^{2}\right)>b_{\alpha}^{2} \\
& \bar{q} \equiv \bar{c}_{\alpha}^{2}-\frac{1}{2}(r-x)
\end{aligned}
$$

and if $t_{0}$ represents the value of $t$ for which $\mu(t)$ is a minimum then

$$
t_{0}=\overline{4 q} \frac{1}{2} x^{-\frac{1}{2}} /\left(1-\bar{q}^{\frac{1}{2}} x^{-\frac{1}{2}}\right) \quad\left(x>\bar{c}_{\alpha}^{2}\right)
$$

because $d \mu / d t=0 ; d^{2} \mu / d t^{2}>0$ for $t=t_{0}$. Moreover

$$
\mu\left(t_{0}\right)=2 x^{\frac{1}{2}} \bar{q}^{\frac{1}{2}}-\bar{q}
$$

is the minimum value of $\mu(t)$. Also

$$
\left.4 \bar{q}^{\frac{1}{2}} x^{-\frac{1}{2}}\right) /\left(1-\bar{q}^{\frac{1}{2}} x^{-\frac{1}{2}}\right)<16 q^{\frac{1}{2}} / x^{\frac{1}{2}} \quad\left(x>2(3)^{\frac{1}{2}} \overline{c_{\alpha}} / 3\right) .
$$

If $\varepsilon_{1} \equiv \frac{1}{2}\left(c_{\alpha}^{2}-b_{\alpha}^{2}\right)$ then

$$
I_{1}=\int_{0}^{16 q^{\frac{1}{2} / x^{\frac{1}{2}}}} \exp (-x t /(t+4)) \exp (-\overline{4 q} / t) \exp \left(-4 \varepsilon_{1} / t\right) t^{-3 / 2}\left(1-e^{-t}\right)^{-\alpha-1} d t .
$$

By the estimate (2.11) this becomes

$$
I_{1} \leqq 2^{\alpha+1} \int_{0}^{16 q^{\frac{1}{2} / x \frac{1}{2}}} \exp (-x t /(t+4)-4 \bar{q} / t) \exp \left(-4 \varepsilon_{1} / t\right) t^{-(\alpha+5 / 2)} d t
$$

for $x>\left(16 c_{\alpha}\right)^{2}$. By (2.12) and (2.13) the above becomes

$$
\begin{aligned}
I_{1} & \leqq \exp \left(-2 x^{\frac{1}{2}} \bar{q}^{\frac{1}{2}}+\bar{q}\right) \int_{0}^{16 q \frac{1}{2} / x \frac{1}{2}} \exp \left(-4 \varepsilon_{1} / t\right) t^{-\alpha-5 / 2} d t \\
& <2^{\alpha+1} \exp \left(-2 x^{\frac{1}{2}} q^{\frac{1}{2}}+\bar{c}_{\alpha}^{2}\right) \Gamma(\alpha+3 / 2)\left(2\left(c_{\alpha}^{2}-b_{\alpha}^{2}\right)\right)^{-\alpha-3 / 2} \\
& \leqq B(\alpha, b) \exp \left(-2 x^{\frac{1}{2}} q^{\frac{1}{2}}\right) \\
& \quad\left(z \in \bar{p}\left(b_{\alpha}\right) ; x>\max \left\{2(3)^{\frac{1}{2}} \overline{c_{\alpha}} / 3 ; \bar{c}_{\alpha}^{2} ;\left(16 c_{\alpha}^{2}\right)^{2}\right\},\right.
\end{aligned}
$$

By (2.9.1), (2.11.1) and the last inequality we have

$$
\begin{aligned}
|f(z)|^{2} \leqq B(\alpha, b) \exp ( & \left.x-2 x^{\frac{1}{2}} q^{\frac{1}{2}}\right) \\
& *\left(z \in \bar{p}\left(b_{\alpha}\right) ; x>\max \left\{15 c_{\alpha}^{2} ; 2(3)^{\frac{1}{2}} \overline{c_{\alpha}} / 3 ; \overline{c_{\alpha}^{2}} \equiv x_{0}\right)\right)
\end{aligned}
$$

for $\alpha>-\frac{1}{2}$.

But since $I_{1}$ and $I_{2}$ are bounded functions of $x$ for $-b_{\alpha}^{2} \leqq x \leqq x_{0}$ we can choose $B(\alpha, b)$ in the last inequality large enough that the inequality holds for all points $z$ in and on the parabola $y^{2}=4 b_{\alpha}^{2}\left(x+b_{\alpha}^{2}\right)$. Hence

$$
|f(z)| \leqq B(\alpha, b) \exp \left(\frac{1}{2} x-|x|^{\frac{1}{2}} q^{\frac{1}{2}}\right) \quad\left(\alpha>-\frac{1}{2}\right)
$$

and for every point $z$ in and on the parabola $y^{2}=4 b_{\alpha}^{2}\left(x+b_{\alpha}^{2}\right)$.

Moreover by (1.3) (2.6) and (2.7) it can be shown that the Laguerre series (1.1) converges absolutely and uniformly in any closed region for 
which $\Re(-z)^{\frac{1}{2}}<d_{\alpha}(\alpha>-1)$ that is, inside the parabola of convergence $y^{2}=4 d_{\alpha}^{2}\left(x+d_{\alpha}^{2}\right)$, and hence represents an analytic function there by [5 p. 74 Theorem 3]. For a proof of this see the dissertation of Evelyn Boyd [1].

This completes the proof of the necessity of Theorem A.

3. The sufficiency of Theorem A. Let $w=u+i v$. Then by the notation $w \in s(b) ; w \in \bar{s}(b)$ where $b>0$ we mean respectively that the point $w$ lies in the open (closed) strip $s(b): v^{2}<b . \bar{s}(b): v^{2} \leqq b$.

If $z=w^{2}$ then

$$
z \in p(b) \rightleftarrows w \in s(b)
$$

by virtue of (1.4) and the fact that

$$
\frac{1}{2}(r-x)=v^{2} .
$$

For the function $h\left(w^{2}\right)$ the following two order conditions are equivalent

$$
\begin{aligned}
& \left|h\left(w^{2}\right)\right|<B_{1}(b) \exp \left(\left(u^{2}-v^{2}\right) / q-k\left|u^{2}-v^{2}\right|^{\frac{1}{2}}\left(b^{2}-v^{2}\right)^{\frac{1}{2}}\right) \\
& \left|h\left(w^{2}\right)\right|<B_{2}(b) \exp \left(u^{2} / q-k|u|\left(b^{2}-v^{2}\right)^{\frac{1}{2}}\right) \\
& \qquad\left(w \in s(b) ; q, k, B_{1}(b), B_{2}(b)>0\right) .
\end{aligned}
$$

LEMma 3.1. We define

$$
g_{\alpha}(w)=w \int_{0}^{1} t^{\alpha} f\left(t w^{2}\right)(1-t)^{-\alpha-\frac{1}{2}} d t \quad\left(w=u+i v ;-1<\alpha<\frac{1}{2}\right) .
$$

We assume the hypothesis of the sufficiency Theorem $A$, then for every $b_{\alpha}, 0 \leqq b_{\alpha}<d_{\alpha}$ there exists a positive number $B(\alpha, b)$ such that

$$
\left|g_{\alpha}(w)\right| \leqq B(\alpha, b) \exp \left(\frac{1}{2} u^{2}-|u|\left(b_{\alpha}^{2}-v^{2}\right)^{\frac{1}{2}}\right) \quad\left(w \in s\left(b_{\alpha}\right) ;-1<\alpha<\frac{1}{2}\right)
$$

and $g_{\alpha}(w)$ is analytic for $w \in s\left(d_{\alpha}\right)$.

Proof. We make the transformation $z=w^{2}$ on the order condition of Theorem A. Then by equivalence relation (3.1) $w \in s\left(b_{\alpha}\right)$ so that in conjunction with (3.2) and (3.5) we conclude

$$
\left|f\left(w^{2}\right)\right| \leqq B(\alpha, b) \exp \left(\frac{1}{2}\left(u^{2}-v^{2}\right)-\left|u^{2}-v^{2}\right|^{\frac{1}{2}}\left(b_{\alpha}^{2}-v^{2}\right)^{\frac{1}{2}}\right)
$$

for $w \in s\left(b_{\alpha}\right)$ and $-1<\alpha$. Then by the equivalence relation (3.3) setting $q=2$ and $k=1$ we get from the above inequality

$$
\left|f\left(w^{2}\right)\right| \leqq B(\alpha, b) \exp \left(\frac{1}{2} u^{2}-|u|\left(b_{\alpha}^{2}-v^{2}\right)^{\frac{1}{2}}\right) \quad\left(w \in s\left(b_{\alpha}\right) ;-1<\alpha\right) .
$$

The change of variable $t=h^{2}$ in the integral of equation (3.4), expresses $\left|g_{\alpha}(w)\right|$ by 


$$
\begin{aligned}
\left|g_{\alpha}(w)\right|=\mid 2 w \int_{0}^{1} h^{2 \alpha+1} f\left(h w^{2}\right) & \left(1-h^{2}\right)^{-\alpha-\frac{1}{2}} d h \mid \\
& \leqq 2|w|\left\{\max _{0 \leq h \leq 1}\left|f\left\{(h w)^{2}\right\}\right|\right\}\left|\int_{0}^{1}\left(h^{2} /\left(1-h^{2}\right)\right)^{\alpha+\frac{1}{2}} d h\right| .
\end{aligned}
$$

The above integral converges for each value of $\alpha$ such that $\alpha<\frac{1}{2}$ and from Pollard's work (see [7 p. 362-363] in particular inequality (3.3)) we conclude that the function outside the integral satisfies the inequality (3.5).

From formula (3.4) by expanding a factor of the integrand into a power series we get

$$
\begin{aligned}
g(w) & =w \int_{0}^{1} t^{\alpha} f\left(t w^{2}\right)\left\{\sum_{n=0}^{\infty}\left(\begin{array}{c}
n+\alpha-\frac{1}{2} \\
n
\end{array}\right) t^{n}\right\} d t \\
& =w \sum_{n=0}^{\infty}\left(\begin{array}{c}
n+\alpha-\frac{1}{2} \\
n
\end{array}\right) \int_{0}^{1} t^{\alpha+n} f\left(t w^{2}\right) d t .
\end{aligned}
$$

Using estimate (2.2) and the maximum modulus theorem on $f\left(t w^{2}\right)$ for the region

$$
\bar{s}\left(d_{\alpha}, D\right): w \in \bar{s}\left(b_{\alpha}\right) ; u^{2}<b_{\alpha}^{2}+D
$$

we get that the above series is in absolute value less then

$$
K_{1} w\left|f\left(z_{0}\right)\right| \sum_{n=0}^{\infty} n^{\alpha-3 / 2}
$$

where $z_{0}$ is a point on the boundary of the region :

$$
\left.\bar{p}_{\alpha} d, D\right): y^{2} \leqq 4 b_{\alpha}^{2}\left(x+b_{\alpha}^{2}\right)
$$$$
x \leqq D
$$

The above series converges for $\alpha<\frac{1}{2}$. This not only justifies the above term by term integration (see [9 p. 45]) but shows that the series representing $g_{\alpha}(w)$ is absolutely and uniformly convergent in the closed region $\bar{s}\left(d_{\alpha}, D\right)$ and hence by [5 p. 73-74] the function $g_{\alpha}(w)$ is analytic if $w$ lies in the simply connected region $s\left(d_{\alpha}\right)$.

Lemma. 3.2. Between Laguerre polynomials of different order there is the following relationship

$\int_{x}^{\infty} e^{-u}(u-x)^{-t} L_{n}^{(\alpha)}(u) d u=e^{-x} L_{n}^{(\alpha+t-1)}(x) \Gamma(1-t) \quad(\alpha>-1 ; t<1 ; x \geqq 0)$,

Proof. From [8 p. 97 formula (5.1.9)] we have

$$
\sum_{n=0}^{\infty} L_{n}^{(\alpha)}(u) s^{n}=(1-s)^{-\alpha-1} \exp (-u s /(1-s))
$$

From which it follows that 


$$
\begin{aligned}
& \int_{x}^{\infty} e^{-u}(u-x)^{-t}\left[\sum_{n=0}^{\infty} L_{n}^{(\alpha)}(u) s^{n}\right] d u \\
& \quad=\int_{x}^{\infty} e^{-u}(u-x)^{-t}(1-s)^{-\alpha-1} \exp (-u s /(1-s)) d u \\
& =(1-s)^{-\alpha-1} e^{-x} \exp (-x s /(1-s)) \int_{0}^{\infty} v^{-t} \exp (-v /(1-s)) d v \\
& =(1-s)^{(-\alpha-t+1)^{-1}} \exp (-x s /(1-s)) e^{-x} \int_{0}^{\infty} e^{-T} T^{(-t+1)}{ }^{-1} d T \\
& =e^{-x} \sum_{n=0}^{\infty} L_{n}^{(\alpha+t-1)}(x) s^{n} \Gamma(1-t) .
\end{aligned}
$$

The last line is obtained by substituting (3.6) in the previous line.

In equation (3.7) we get the third line from the second by change of variable $u-x=v$ and the fourth from the third by $T=v /(1-s)$.

That the integrand in the first line of (3.7) is absolutely and uniformly convergent (fixed $\alpha>-1$; fixed $t,|s|<1 ; 0 \leqq x<D_{1} \leqq u \leqq D_{2}$ ) we can show by comparing it with the series $C_{\alpha} M \sum_{n=0}^{\infty} n^{\alpha} s^{n}$ which can be done by using estimates (2.7) and (2.8). Thus the integrand of (3.15) can be integrated term by term over the interval $0<D_{1} \leqq u \leqq D_{2}$. We obtain

$$
\begin{aligned}
0 & \leqq \sum_{n=0}^{\infty} \int_{x}^{\infty} e^{-u}(u-x)^{-t}\left|L_{n}^{(\alpha)}(u)\right| s^{n} d u \\
& \leqq c_{\alpha} e^{-\frac{1}{2} x} 2^{1-t} \Gamma(1-t) \sum_{n=0}^{\infty} n^{\alpha} s^{n}
\end{aligned}
$$

by using estimates (2.7) and (2.8) on the first series in the above and then making the change of variable $\frac{1}{2}(u-x)=p$. Thus the above series converges and since the series of (3.7) is uniformly convergent by [9] p. 45 can integrate the first series of (3.7) term by term:

$$
\begin{aligned}
\sum_{n=0}^{\infty} s^{n} \int_{x}^{\infty} e^{-u}(u-s)^{-t} L_{n}^{(\alpha)}(u) d u \\
\quad=\int_{x}^{\infty} e^{-u}(u-x)^{-t}\left[\sum_{n=0}^{\infty} L_{n}^{(\alpha)}(u) s^{n}\right] d u=e^{-x} \sum_{n=0}^{\infty} L_{n}^{(\alpha+t-1)}(x) s^{n} \Gamma(1-t) .
\end{aligned}
$$

Equating the coefficients of the two power series in $s$, we get Lemma 3.2. If in Lemma 3.2 we set $\alpha=\frac{1}{2}$ and if in the resulting equation we set $\alpha=t-\frac{1}{2}<\frac{1}{2}$ (since $t<1$ ) we obtain

$$
\begin{array}{r}
\int_{x}^{\infty} e^{-u}(u-x)^{-\alpha-\frac{1}{2}} L_{n}\left(\frac{1}{2}\right)(u) d u=\Gamma\left(-\alpha+\frac{1}{2}\right) e^{-x} L_{n}^{(\alpha)}(x) \\
\left(-1<\alpha<\frac{1}{2} ; x \geqq 0\right) .
\end{array}
$$

Lemma 3.3 (Hille's lemma). By Lemma 3.1 if $f(z)$ satisfies the order condition (1.5) of Theorem A and is analytic then $g_{\alpha}(w)$ is analytic and satisfies Hille's condition for an analytic function to possess a convergent 
Hermitian series, i.e. condition (3.5) (see [4 p. 81 Theorem 1]). Hence we may write

$$
g_{\alpha}(w)=\sum_{n=0}^{\infty}\left(2^{n} n ! \pi^{\frac{1}{2}}\right)^{-\frac{1}{2}} f_{n} H_{n}(w)
$$

where

$$
\left(2^{2 n+1}(2 n+1) ! \pi^{\frac{1}{2}}\right)^{\frac{1}{2}} f_{2 n+1}=\int_{-\infty}^{\infty} \exp \left(-q^{2}\right) H_{2 n+1}(q) g_{\alpha}(q) d q .
$$

Pick any $b<d$, where $2 d$ is the width of the strip of convergence of the above series. Select $\bar{b}$ such that $b<\bar{b}<d$. Then by $[4$ p. 90] we have that there exists a positive number $B(b)$ such that

$$
\left|f_{2 n+1}\right|<B(b) \exp \left(-\bar{b}(4 n+3)^{\frac{1}{2}}\right) .
$$

Lemma 3.4. Let $d>0$ and suppose $f(z)$ is analytic for $z \in p(d)$. Moreover suppose for every $b$ such that $0 \leqq b<d$ there exists a positive number $B(\alpha, b)$ such that

$$
|f(z)| \leqq B(\alpha, b) \exp \left(\frac{1}{2} x-|x|^{\frac{1}{2}}\left(b^{2}-\frac{1}{2}[r-x]\right)^{\frac{1}{2}}\right) \quad(z \in p(b)),
$$

then

$$
\left|a_{n}^{\alpha}\right| \leqq B(b) \exp \left(-2 n^{\left.\frac{1}{2} b\right)} \quad\left(-1<\alpha<\frac{1}{2}\right)\right.
$$

Proof. By estimate (2.7) and inequality (3.11) the following integral converges

$$
\begin{aligned}
a_{n}^{(\alpha)}= & \left\{\left(\begin{array}{c}
n+\alpha \\
n
\end{array}\right) \Gamma(\alpha+1)\right\}^{-1} \int_{0}^{\infty} t^{\alpha} e^{-t} f(t) L_{n}^{(\alpha)}(t) d t \\
= & \left\{\left(\begin{array}{c}
n+\alpha \\
n
\end{array}\right) \Gamma(\alpha+1) \Gamma\left(-\alpha+\frac{1}{2}\right)\right\} \int_{0}^{\infty} t^{\alpha} f(t) d t \\
& \times \int_{t}^{\infty} e^{-s} L_{n}\left(\frac{1}{2}\right)(s)(s-t)^{-\alpha-\frac{1}{2}} d s \quad(b y \quad(3.8)) \\
= & \left\{\left(\begin{array}{c}
n+\alpha \\
n
\end{array}\right) \Gamma(\alpha+1) \Gamma^{\prime}\left(-\alpha+\frac{1}{2}\right)\right\}^{-1} \int_{0}^{\infty} e^{-s} L_{n}\left(\frac{1}{2}\right)(s) d s \\
& \times \int_{0}^{s} t^{\alpha} f(t)(s-t)^{-\alpha-\frac{1}{2}} d t
\end{aligned}
$$

The reversal of integration will be justified later. Making two successive changes of variable $t=p s ; s=q^{2}$ we obtain

$$
\begin{aligned}
a_{n}^{\alpha} & =\left\{\left(\begin{array}{c}
n+\alpha \\
n
\end{array}\right) \Gamma(\alpha+1) \Gamma\left(-\alpha+\frac{1}{2}\right)\right\}^{-1} \\
& \times \int_{0}^{\infty} 2 \exp \left(-q^{2}\right) L_{n}\left(\frac{1}{2}\right)\left(q^{2}\right) q^{2} d q \int_{0}^{1} p^{\alpha} f\left(p q^{2}\right)(1-p)^{-\alpha-\frac{1}{2}} d p
\end{aligned}
$$




$$
\begin{aligned}
& =2\left\{\left(\begin{array}{c}
n+\alpha \\
n
\end{array}\right) \Gamma(\alpha+1) \Gamma\left(-\alpha+\frac{1}{2}\right)(-1)^{n} 2^{2 n+1} n !\right\}^{-1} \\
& \times \int_{0}^{\infty} \exp \left(-q^{2}\right) H_{2 n+1}(q) g_{\alpha}(q) d q .
\end{aligned}
$$

From [8 p. 102 formula (5.6.1)] we have the formula relating Laguerre and Hermite polynomials

$$
q L_{n}\left(\frac{1}{2}\right)\left(q^{2}\right)=\left\{(-1)^{n} 2^{2 n+1} n !\right\}^{-1} H_{2 n+1}(q)
$$

By this relationship and (3.4) the above expression for $a_{n}^{(\alpha)}$ becomes

$$
\begin{aligned}
a_{n}^{(\alpha)}= & \left\{\left(\begin{array}{c}
n+\alpha \\
n
\end{array}\right) \Gamma(\alpha+1) \Gamma^{\top}\left(-\alpha+\frac{1}{2}\right)(-1)^{n} 2^{2 n+1} n !\right\}^{-1} \\
& \times \int_{-\infty}^{\infty} \exp \left(-q^{2}\right) H_{2 n+1}(q) g_{\alpha}(q) d q
\end{aligned}
$$

since the integrand is an even function. By (3.9)

$$
\begin{aligned}
\alpha_{n}^{\alpha}= & \left\{2^{2 n+1}(2 n+1) ! \pi^{\frac{1}{2}}\right\}^{\frac{1}{2}} f_{2 n+1}\left\{\left(\begin{array}{c}
n+\alpha \\
n
\end{array}\right) \Gamma(\alpha+1) \Gamma\left(-\alpha+\frac{1}{2}\right)(-1)^{n} 2^{2 n+1} n !\right\}^{-1} \\
\leqq & \left\{2^{2 n+1}(2 n+1) ! \pi^{\frac{1}{2}}\right\}^{\frac{1}{2}} B(\bar{b}) \\
& \times\left\{\left(\begin{array}{c}
n+\alpha \\
n
\end{array}\right) \Gamma(\alpha+1) \Gamma\left(-\alpha+\frac{1}{2}\right)(-1)^{n} 2^{2 n+1} n !\right\}^{-1} \exp \left(-\bar{b}(4 n+3)^{\frac{1}{2}}\right) .
\end{aligned}
$$

This follows from (3.10). Using Stirling's theorem (see [9 formula (4) p. 58])

$$
n ! \sim(2 \pi)^{\frac{1}{2}} n^{n+\frac{1}{2}} e^{-n}
$$

and the relation $\Gamma(n)=(n-1) \Gamma(n-1)$ as well as the estimate (2.2) we get from the above inequality

$$
\begin{aligned}
a_{n}^{\alpha} & \leqq K_{1} n^{-\alpha+\frac{1}{4}} 2 \Gamma\left(-\alpha+\frac{1}{2}\right) \exp \left(-\bar{b}(4 n+3)^{\frac{1}{2}}\right) \\
& \leqq B(\bar{b}) \exp \left\{\left(-\alpha+\frac{1}{4}\right) \log n+2 n^{\frac{1}{2}}(b-\bar{b})\right\} \exp \left(-2 b n^{\frac{1}{2}}\right) \\
& <B(\bar{b}) \exp \left(-2 b n^{\frac{1}{2}}\right) \quad\left(-1<\alpha<\frac{1}{2}\right)
\end{aligned}
$$

since $\bar{b}>b$. We can justify the above interchange of order of integration by proving that the integral is absolutely convergent. (To use [9 p. 55] consider a function which is zero over part of the rectangular region).

Since $L_{n}^{\frac{1}{2}(s)}$ is a polynomial of degree $n$ we have for a fixed $n$

$$
\left|e^{-\frac{1}{2} s} L_{n}\left(\frac{1}{2}\right)(s)\right|<c \quad(0 \leqq s<\infty) .
$$

From the above estimate and (3.11) the integral in the second line of (3.13) is in absolute value less than 


$$
B(\alpha, b) \int_{0}^{\infty} t^{x} \exp \left(\frac{1}{2} t-t^{\frac{1}{2}} b\right) d t \int_{t}^{\infty} e^{-\frac{1}{2} s}(s-t)^{-\alpha-\frac{1}{2}} d s \quad\left(-1<\alpha<\frac{1}{2}\right) .
$$

By change of variable $s-t=2 p$ on the inside integral and then $q=t^{\frac{1}{2}} b$ on the outside integral this becomes

$$
\begin{array}{r}
B(\alpha, b) 2^{-\alpha+3 / 2} \Gamma\left(-\alpha+\frac{1}{2}\right) b^{-2 \gamma-2} \int_{0}^{\infty} q^{2 \alpha+2-1} e^{-q} d q=B(\alpha, b) \Gamma(2 \alpha+2) \\
\left(-1<\alpha<\frac{1}{2}\right) .
\end{array}
$$

Conclusion. Since by hypothesis $f(z)$ is analytic for $z \in p\left(d_{\alpha}\right)$ the function $f(x)$ is continuous for $x \geqq 0$ and hence integrable for $0 \leqq x \leqq$ $D(D>0)$. Moreover since by hypothesis inequality (1.5) holds we have $|f(x)|<A e^{x / 2}$ for $x \geqq 0$. Then by a theorem of Caton and Hille [2 p. 227] series (1.1) is Abel summable to $f(x)$ for almost all $x$, including the points of continuity and hence in this case for all points $x$ for which $x \geqq 0$.

By virtue of (3.12) and (2.6) it follows that the limit (1.3) exists that is $d_{\iota}>0$ and series (1.1) converges for $z \in p\left(d_{\alpha}\right)$ and for $-1<\alpha<\frac{1}{2}$. We now want to generalize this result to the condition $-1<\alpha$.

From (1.2) and [8 p. 98 formula (5.1.14)] and [9 p. 55 formula (2)] we have $a_{n}^{(\alpha+1)}=a_{n}^{(\alpha)}-a_{n+1}^{(\alpha)}$. Let

$$
s_{n}^{\alpha}=\sum_{k=0}^{n} L_{n}^{(\alpha)}(z) \quad(n=0,1,2, \cdots)
$$

then

$$
\sum_{k=0}^{n} a_{k}^{(\alpha)} L_{k}^{(\alpha)}(z)=\sum_{k=0}^{n-1}\left(a_{k}^{(\alpha)}-a_{k+1}^{(\alpha)}\right) s_{k}^{\alpha}+a_{n}^{(\alpha)} s_{n}^{\alpha}=\sum_{k=0}^{n-1} a_{n}^{(\alpha+1)} L_{k}^{(\alpha+1)}(z)+a_{n}^{\alpha} L_{n}^{(\alpha+1)}(z) .
$$

By (1.3) (2.6) and (2.7) we have for a fixed $z_{0},\left|a_{n}^{(\alpha)} L_{n}^{(\alpha+1)}\left(z_{0}\right)\right| \rightarrow 0$ for $n \rightarrow \infty$ for $z_{\jmath} \in p\left(d_{\alpha}\right)$ and for $-1<\alpha<\frac{1}{2}$. Hence

$$
\sum_{n=0}^{\infty} a_{n}^{(\alpha)} L_{n}^{(\alpha)}(z)=\sum_{n=0}^{\infty} a_{n}^{(\alpha+1)} L_{n}^{(\alpha+1)}(z) \quad\left(z \in p\left(d^{x}\right) ;-1<\alpha<\frac{1}{2}\right) .
$$

Hence, by mathematical induction, the range of $\alpha$ for which the series (1.1) converges has been extended to $-1<\alpha$.

Since the Abel sum of series (1.1) is $f(x)$ for $x \geqq 0$ and also the same as the Cauchy sum, the Cauchy sum of series (1.1) is $f(x)$ for $x \geqq 0$ and $-1<\alpha$.

We remarked at the end of $\S 2$ that series (1.1) is an analytic function inside its parabola of convergence. Hence by the identity theorem for analytic functions (see [5 p. 87]) since both $f(z)$ and series (1.1) are analytic for $z \in p\left(d_{c}\right)$ and identical along the real axis they must be identical for $z \in p\left(d_{\alpha}\right)$ that is inside their common region of analyticity which is the parabola of convergence of the series: $y^{2}=4 b_{\alpha}^{2}\left(x+b_{\alpha}^{2}\right)$ $\left(0 \leqq b_{\alpha}<d_{\alpha}\right)$. 
This completes the proof of Theorem A.

4. The equivalence of Theorems A and B. We now note that Theorem $A$ is equivalent to a generalization of Pollard's Theorem A [7]. We state the generalization as follows.

THEOREM B. In order that $g(w)$ possess a Laguerre series of order alpha $(\alpha>-1)$ such that

$$
f(z)=f\left(w^{2}\right)=g(w)=\sum_{n=0}^{\infty} a_{n}^{(\alpha)} L_{n}^{(\alpha)}\left(w^{2}\right) \quad\left(w \in s\left(d_{\alpha}\right) ; d_{\alpha}>0\right)
$$

where $a_{n}^{(\alpha)}$ is given by (1.2) it is necessary and sufficent that $g(w)$ be analytic and even for $w \in s\left(d_{\alpha}\right)$ and that to every $b_{\alpha}$ with $0 \leqq b_{\alpha}<d_{x}$ there correspond a positive number $B(\alpha, b)$ such that

$$
|g(w)| \leqq B(\alpha, b) \exp \left(\frac{1}{2} u^{2}-|v|\left(b^{2}-v^{2}\right)^{\frac{1}{2}}\right) \quad\left(w \in s\left(d_{\alpha}\right), \alpha>-1\right) .
$$

THEOREM C. Theorems A and B are equivalent.

Proof. For

$$
z=w^{2}
$$

the equation of Theorem $\mathrm{B}$ becomes

$$
f(z)=\sum_{n=0}^{\infty} \alpha_{n}^{(\alpha)} L_{n}^{(\alpha)}(z)
$$

and conversely by (3.1).

Since $f(z)=f\left(w^{2}\right)=g(w)$ the function $g(w)$ will be analytic if $f(z)$ is. Moreover since $f(z)=g\left(z^{\frac{1}{2}}\right)$ we get the converse by the same reasoning except at $z=0$, since $z^{\frac{1}{2}}$ is analytic except at the origin. In a neighborhood of $w=0$ we have since $g(w)$ is even and analytic

$$
f(z)=f\left(w^{2}\right)=g(w)=\sum_{n=0}^{\infty} a_{n} w_{n}^{2 n}=\sum_{n=0}^{\infty} a_{n} z^{n}
$$

so that $f(z)$ is analytic at $z=0$ also.

Applying the transformations (4.2) and (3.2) and the equivalence relations (3.3) to the inequalities (4.1) and (1.5) we get their equivalence because of (3.1).

\section{REFERENCES}

1. Evelyn Boyd, On Laguerre series in the complex domain, Dissertation 1949, Yale University.

2. W. B. Caton and E. Hille, Laguerre polynomials and Laplace integrals, Duke Math. J. 12 (1945), 217-242. 
3. E. Hille, Contributions to the theory of Hermitian series, Duke Math. J., 5 (1939), 875-936.

4. - Contributions to the theory of Hermitian series, II. The representation problem, Trans. Amer. Math. Soc., 47 (1940) 80-94.

5. K. Knopp, The theory of functions, Dover. Vol. I, (1945).

6. O. Perron, Über das Verhalten einer ausgearieten hypergeometrischen Reihe bei unbegrentztem Wachstum eines Parameters, J. Reine Angew. Math. 151 (1921), 63-78.

7. H. Pollard, Representation of an analytic function by a Laguerre series, Ann. of Math. 48 (series 2) (1947), 358-365.

8. G. Szegö, Orthogonal polynomials, Amer, Math. Soc. Colloq. Publ. XXIII (1939).

9. E. C. Titchmarsh, The theory of functions, Oxford. Second edition, 1939.

10. G. Watson, A treatise on the theory of Bessel functions, Cambridge. Second edition, 1944.

11. S. Wigert, Contributions a la theorie des polynomes d'Abel-Laguerre, Ark. Math. 15, (1921), No. 25.

12. A. Zygmund, Trigonometrical series. Warsaw (1935).

Thiel College 



\section{PACIFIC JOURNAL OF MATHEMATICS}

\section{EDITORS}

David Gilbarg

Stanford University

Stanford, California

R. A. Beaumont

University of Washington

Seattle 5, Washington

\author{
A. L. Whiteman
}

University of Southern California

Los Angeles 7, California

E. G. Straus

University of California

Los Angeles 24, California

\section{ASSOCIATE EDITORS}

\author{
E. F. BECKENBACH \\ C. E. BURGESS \\ M. HALL \\ E. HEWITT
}

\author{
A. HORN \\ V. GANAPATHY IYER \\ R. D. JAMES \\ M. S. KNEBELMAN
}

L. NACHBIN
I. NIVEN
T. G. OSTROM
H. L. ROYDEN

M. M. SCHIFFER

G. SZEKERES

F. WOLF

K. YOSIDA

\section{SUPPORTING INSTITUTIONS}

\author{
UNIVERSITY OF BRITISH COLUMBIA \\ CALIFORNIA INSTITUTE OF TECHNOLOGY \\ UNIVERSITY OF CALIFORNIA \\ MONTANA STATE UNIVERSITY \\ UNIVERSITY OF NEVADA \\ OREGON STATE COLLEGE \\ UNIVERSITY OF OREGON \\ UNIVERSITY OF SOUTHERN CALIFORNIA
}

\author{
STANFORD UNIVERSITY \\ UNIVERSITY OF UTAH \\ WASHINGTON STATE COLLEGE \\ UNIVERSITY OF WASHINGTON \\ AMERICAN MATHEMATICAL SOCIETY \\ CALIFORNIA RESEARCH CORPORATION \\ HUGHES AIRCRAFT COMPANY \\ THE RAMO-WOOLDRIDGE CORPORATION
}

Mathematical papers intended for publication in the Pacific Journal of Mathematics should be typewritten (double spaced), and the author should keep a complete copy. Manuscripts may be sent to any of the editors. All other communications to the editors should be addressed to the managing editor, E. G. Straus at the University of California, Los Angeles 24, California.

50 reprints per author of each article are furnished free of charge; additional copies may be obtained at cost in multiples of 50 .

The Pacific Journal of Mathematics is published quarterly, in March, June, September, and December. The price per volume (4 numbers) is $\$ 12.00$; single issues, $\$ 3.50$. Back numbers are available. Special price to individual faculty members of supporting institutions and to individual members of the American Mathematical Society: $\$ 4.00$ per volume; single issues, $\$ 1.25$.

Subscriptions, orders for back numbers, and changes of address should be sent to Pacific Journal of Mathematics, 2120 Oxford Street, Berkeley 4, California.

Printed at Kokusai Bunken Insatsusha (International Academic Printing Co., Ltd.), No. 6, 2-chome, Fujimi-cho, Chiyoda-ku, Tokyo, Japan.

\section{PUBLISHED BY PACIFIC JOURNAL OF MATHEMATICS, A NON-PROFIT CORPORATION}

The Supporting Institutions listed above contribute to the cost of publication of this Journal, but they are not owners or publishers and have no responsibility for its content or policies. 


\section{Pacific Journal of Mathematics}

\section{Vol. 8, No. 3 \\ May, 1958}

Michael Israel Aissen, A set function defined for convex plane domaines... . 383

Robert Ellis, Distal transformation groups ................... 401

Ciprian Foias, On a commutative extension of a commutative Banach algebra ....................................... 407

Jerry William Gaddum, Linear inequalities and quadratic forms ......... 411

Allen A. Goldstein and Elliott Ward Cheney, Jr., A finite algorithm for the solution of consistent linear equations and inequalities and for the Tchebycheff approximation of inconsistent linear equations...........

William L. Hart and T. S. Motzkin, Proof of the fundamental theorem on implicit functions by use of composite gradient corrections .......... 429

Henry Berge Helson, Conjugate series and a theorem of Paley .......... 437

Wu-Chung Hsiang, Abelian groups characterized by their independent subsets....................................... 447

John W. Lamperti, On the isometries of certain function-spaces ........ 459

Karel DeLeeuw and Walter Rudin, Extreme points and extremum problems

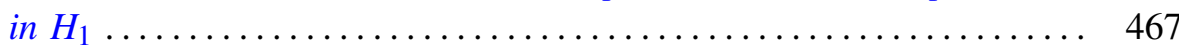

Eugene Lukacs, Some extensions of a theorem of Marcinkiewicz........ 487

George W. Mackey, Multiplicity free representations of finite groups ..... 503

Eben Matlis, Injective modules over Noetherian rings ............. 511

John William Neuberger, Continuous products and nonlinear integral equations

Lawrence Edward Payne and Hans F. Weinberger, New bounds for solutions of second order elliptic partial differential equations...

William T. Reid, A Prüfer transformation for differential systems ........ 575

Howard L. Rolf, The free lattice generated by a set of chains ...

K. M. Saksena, Inversion and representation theorems for a generalized

Laplace integral....................................... 597

Daniel Shanks, Two theorems of Gauss......................... 609

Paul Slepian, On the Lebesgue area of a doubled map ............... 613

Otto Szász and Nelson Paul Yeardley, Jr., The representation of an analytic function by general Laguerre series . ..................... 621

Alan C. Woods, On two-dimensional convex bodies ................. 635 